\title{
Systematic Derivation of an Australian Standard for Tall Man Lettering to Distinguish Similar Drug Names
}

\author{
Running title: Derivation of a Tall Man Lettering Standard
}

Lynne Emmerton, BPharm(Hons) PhD MPS ${ }^{1 *}$, Associate Professor

Mariam FS Rizk, BPharm(Hons) ${ }^{1}$, MBBS candidate

Graham Bedford, BA, DipLaw, Policy Team Director

Daniel Lalor, BPharm(Hons) MBA (Health Management) ${ }^{3}$, Manager, Medication Safety

1 The University of Queensland, Brisbane, Australia

2 Australian Commission on Safety and Quality in Health Care, Sydney, Australia

3 Clinical Excellence Commission, Sydney, Australia (now The Canberra Hospital, Canberra, Australia)

*Author for correspondence (and current affiliation):

Assoc Prof Lynne Emmerton

School of Pharmacy

Curtin University

GPO Box U1987

Perth WA 6845

Australia

Tel: $\quad+61892667352$

Fax: $\quad+61892662769$

Email: lynne.emmerton@curtin.edu.au

Keywords: Tall Man, errors, confusion, look-alike sound-alike, standard, drug Word count: 2201 (excluding title page, abstract, references, figures, tables) 


\section{Systematic Derivation of an Australian Standard for Tall Man Lettering to Distinguish Similar Drug Names}

\section{ABSTRACT}

Rationale, Aims and Objectives: Confusion between similar drug names can cause harmful medication errors. Similar drug names can be visually differentiated using a typographical technique known as Tall Man lettering. While international conventions exist to derive Tall Man representation for drug names, there has been no national standard developed in Australia. This paper describes the derivation of a risk-based, standardised approach for use of Tall Man lettering in Australia, and known as National Tall Man Lettering.

Method: A three-stage approach was applied. An Australian list of similar drug names was systematically compiled from the literature and clinical error reports. Secondly, drug name pairs were prioritised using a risk matrix based on the likelihood of name confusion (a fourcomponent score) $v s$ consensus ratings of the potential severity of the confusion by 31 expert reviewers. The mid-type Tall Man convention was then applied to derive the typography for the highest priority drug pair names.

Results: Of 250 pairs of confusable Australian drug names, comprising 341 discrete names, 35 pairs were identified by the matrix as an 'extreme' risk if confused. The Mid-type Tall Man convention was successfully applied to the majority of the prioritised drugs; some adaption of the convention was required.

Conclusion: This systematic process for identification of confusable drug names and associated risk, followed by application of a convention for Tall Man lettering, has produced a standard now endorsed for use in clinical settings in Australia. Periodic updating is recommended to accommodate new drug names and error reports. 


\section{INTRODUCTION}

The potential for drug name similarity to cause medication errors is widely acknowledged by health care professionals[1], pharmaceutical manufacturers[2], safety agencies[3], professional indemnity insurers[4] and regulators[5,6]. It is approximated that one-quarter of medication-related incidents voluntarily reported in the United States of America (USA) are caused by drug name confusion[7].

Research in Australia[8] and the USA[9] has identified numerous factors contributing to drug name confusion, including similarity in the appearance of the drug names (orthography), sound of the drug names (phonology), strengths of the products available, routes of administration, dosage forms of the products, and indications for use. Screening for the similarity of drug names pre-marketing is the best harm-minimisation strategy[10]. However, confusable drug names may only become evident in practice via error and near-miss reports, whereupon clinicians are referred to published lists and warnings[3,7,11-13].

Researchers in cognitive psychology, linguistics and computer science have developed measures to quantify the orthographic similarity of two drug names[14-16]. The BI-SIM measure reportedly has the greatest accuracy when predicting drug name confusion[14]. This measure places scoring emphasis on similarities at the beginning of pairs of drug names, assuming that the risk of confusing two names will be increased if they appear in close proximity in a list (e.g. on a computer/device screen) or if products are stored alphabetically in close proximity. BI-SIM scores range from 0.00-1.00. 
One initiative to minimise drug confusion errors in clinical practice is the use of Tall Man lettering to distinguish similar drug names[8]. Tall Man lettering uses selective capitalisation to highlight differences among orthographically similar drug names[17-19]. Acceptability of this technique has been demonstrated in an Australian hospital, with researchers calling for a national list of "look-alike sound-alike" drugs using Tall Man typography[20].

Overuse of Tall Man lettering may reduce its effectiveness[18]; as such, it should be reserved for confusable drugs with the greatest potential to cause patient harm, identified through a transparent, reproducible risk-assessment process and based on the best-available evidence. An evaluation of various Tall Man conventions concluded that the Mid-Tall Man rule was the most effective and most easily applied systematically[21].

This paper reports the development of an evidence-based Australian standard for Tall Man nomenclature, to promote consistency in application of the technique for clinicians, software vendors, regulators and the pharmaceutical industry. Our objectives were to:

1. Develop a comprehensive list of confusable drug name pairs

2. Prioritise confusable drug name pairs for Tall Man representation

3. Apply Tall Man nomenclature to the prioritised drug name pairs.

\section{METHODS}

\section{Development of a Comprehensive List of Confusable Drug Name Pairs}

A list of confusable drug names, including generic and brand names, was compiled from: 
- A preliminary list of medications[22] of confusable drugs published in the international literature, and confirmed as marketed brand or generic drug names in Australia

- Websites of five medication safety agencies spanning the USA, Europe and Australia

- Jurisdictional databases of incidents that involved drug name confusion

- Online warnings and alerts published in the 'grey' literature, including incidence reports of drug name confusion from Pharmaceutical Defence Limited, the key pharmacists' indemnity body in Australia.

\section{Prioritisation of Confusable Drug Names for Tall Man Representation}

Confusable drugs were prioritised via a risk matrix (Figure 1) derived using principles outlined by the National Patient Safety Agency in the United Kingdom[23] and the National Coordinating Council for Medication Error Reporting and Prevention in the USA[24]. The matrix comprised two parameters:

1. The 'likelihood of confusion' of the two drugs of interest, assessed using a scoring prototype developed by a multi-disciplinary reference group. Scoring was undertaken by one author (DL) and reviewed by the reference group. To account for factors contributing to confusion between similar drug products, this score arbitrarily comprised:

- Name similarity, as calculated using BI-SIM, using an online calculator[25] (70\%)

- Strength similarity: scored as 0,10 or $20(20 \%)$

- Administration route similarity: scored as $0,2.5$ or $5(5 \%)$

- Dosage form similarity: scored as $0,2.5$ or $5(5 \%)$.

The subsequent score was out of 100. Scores were broken into quintiles for application of the risk matrix. 
2. The potential severity, or consequence, of confusion between similarly-named drugs, based on clinical judgement of the hypothetical error, and also presented as quintiles in the matrix. Two assumptions were applied: that the exposure to the wrong drug was short term (i.e. that the error was detected within one week), and that the person receiving the wrong drug was otherwise healthy. As confusion between two drug names can occur in either of two directions (i.e. drug $\mathrm{A}$ intended but $\mathrm{B}$ given, or drug $\mathrm{B}$ intended and $\mathrm{A}$ given), severity was conservatively based on the direction with the greater potential for harm. The potential severity rating also considered:

- Drug characteristics: attention was drawn to high-alert drugs such as concentrated electrolytes, insulin, anticoagulants, opioids and cytotoxics, those with known major drug-drug interactions, narrow-therapeutic-index drugs, those with high prevalence of allergic reaction, and drugs with time-critical administration

- The number of doses of the wrong drug that would cause harm.

Severity assessment used two-stage expert review. The 31 experts comprised clinical pharmacists with varying roles $(n=26$, including author $\mathrm{DL})$, clinical pharmacologists $(n=2)$, safety officers with nursing backgrounds $(n=2)$ and a nurse unit manager. All were provided instructions (available on request) for completing the severity assessment. The first stage required rating of 20 randomly-selected name pairs by all reviewers to test the consistency of their ratings, calculated as an intra-class correlation $(\alpha)$ using $\operatorname{SPSS}^{\circledR}$. The second stage involved independent, online rating in 10 groups of three reviewers (excluding author, with each group randomly allocated 23 name pairs. Two local groups met and discussed their evaluation, reaching consensus on the severity before submitting their results. Where agreement between reviewers was not unanimous, ratings were based on the majority view. 


\section{Application of Tall Man Nomenclature}

The typography rules of the Mid-Tall Man convention[21] (Figure 2) were applied to the drug name pairs that met criteria for 'extreme' or 'high' risk in the risk matrix. Limitations of this convention were anticipated for larger groups of confusable drug names, such as 10 cephalosporin antibiotics commencing with 'cef' or 'ceph'. In these instances, the name pair with the greatest risk rating (from Figure 1) was used as the index pair. Where there was no natural or logical grouping, Mid-Tall Man lettering was applied first to the name pair (or natural grouping) that carried the highest risk, and then subsequent pairs. Other adaptations of the convention for groups of drug names are illustrated in Figure 3.

Drug pairs that were identified as confusable, but not sharing sufficient orthographic similarity, were excluded from this Tall Man convention. An example is Fungizone ${ }^{\circledR} v s$ Ambisome $^{\circledR}$, whose confusion is likely due to their being different formulations of the same active ingredient, amphotericin B.

Conventions for generic drug names to be presented in all lower case and proprietary names to be presented as proper nouns (i.e. with a capital first letter followed by lower case) were ignored, in contrast to other applications of Tall Man nomenclature[26]. An example is the proprietary products, ciprAMIL ${ }^{\circledR}$ and ciprOXIN ${ }^{\circledR}$.

While brand names, and their presentation, are subject to trademark, legal advice was that such trademarks generally allow the trademark owner flexibility in how the word is presented. These trademarks were not seen as inhibiting the application of Tall Man lettering to proprietary names. 


\section{RESULTS}

The confusable drug names list, available on request, comprised 250 pairs of Australian drugs (341 discrete names): 156 generic names and 185 proprietary (brand) names. Composite 'likelihood of confusion' scores for the 250 drug pairs ranged from 15.0 to 82.5 (out of 100), with a mean of $48.1(\operatorname{std} \operatorname{dev}=12.1)$. The distribution of this variable was determined to be normal using the Kolmogorov-Smirnov test $(\mathrm{p}=0.724)$.

The severity ratings across 20 common pairs by 31 reviewers demonstrated intra-class correlation of $\alpha=0.961$ (95\% confidence interval 0.931-0.982, $\mathrm{p}<0.001)$. Twenty-eight of the 31 reviewers completed the severity rating for the further 23 drug name pairs, with a minimum of three reviewers rating each item. Majority agreement on the severity rating was reached for over $85 \%$ of drug pairs.

Of the 250 drug name pairs identified from available sources as confusable, 35 met criteria of 'extreme' risk of confusability, based on the risk matrix. These comprised 64 discrete drug names. The final list comprises 87 drugs in routine use, and separate lists of 14 drugs used in Oncology, nine cephalosporin antibiotics ('cef-' or 'ceph-'), four sulfonylurea hypoglycaemic agents ('gli-'), four benzodiazepines ('-azepam') and four psychotropic drugs ('-oxetine' or 'oxamine'). 


\section{DISCUSSION}

Our project was a purposeful approach towards risk minimisation in clinical practice, producing a standard for the strategic use of a typographic technique to distinguish similar drug names. The strength and novelty of this work lies in the systematic processes undertaken, firstly, to identify locally-available drugs with measureable name similarity and clinically significant consequences if erroneously switched, and secondly, to apply an accepted convention for Tall Man representation of these names. This drew on international literature reporting drugs associated with errors of confusion, measurement of orthographical similarity, and risk assessment. Further to the body of research on Tall Man typography, we developed solutions to address challenges in the application of this technique, in particular, the management of groups (rather than pairs) of similar drug names and speciallytrademarked drug names.

The resulting Australian National Tall Man Lettering ${ }^{1}$ is an explanatory document appended with a list of Australian-marketed drugs represented in Tall Man typography. Importantly, drugs in this list are limited to those with significant name similarity and those likely to cause significant harm if selected in error, to minimise overuse of the technique that may lead to 'alert fatigue'[8]. The practical limitation of this approach is the exclusion of drug names that may indeed be prone to errors of confusion but with a low risk of patient harm (such as dopamine and dobutamine). Further, the Tall Man convention is not suitable for drugs that may be confused for reasons other than similarity in their names. In both cases, best-practice interventions, such as use of barcode scanners during dispensing[27-29] and administration[30], and separation of confusable medicines in storage[8], are recommended.

\footnotetext{
${ }^{1}$ Published in full at http://www.safetyandquality.gov.au/wp-content/uploads/2012/02/National-Tall-ManLettering-Report-and-final-list1.pdf
} 
Pre-market testing of potentially-confusable drug names may also minimise selection errors by health professionals.

The key assumption behind the compilation of the list of confusable drug names is that errors relating to drug selection have indeed been reported and published[31-34]. Under-reporting of incidents, and particularly near-misses, is common. It is also possible that reporting systems contain relevant data that are not readily retrievable. Additionally, newer drugs with confusable names may pose a significant risk to patient safety, but may not have been marketed long enough for this risk to have become apparent.

Our application of a similarity score and clinical judgement as two dimensions of a risk matrix is novel. Additional clinical considerations, such as the likelihood that the error would be detected and the frequency with which the error is likely to occur, would enhance the risk assessment; however, these are not easily measured, and the subjectivity would add burden to the clinical judgement. The potential consequence of confusion between two drug products is already, by necessity, a subjective measure, and difficult to judge, as factors such as the duration of exposure to the wrong drug and the patient's co-morbidities, other medicines and overall wellbeing will impact significantly on the outcome. Our use of expert consensus for this variable is a strength of our method, with strong correlation between reviewers' scores.

The limitations in identifying confusable drug names, and in prioritising these drugs for Tall Man representation, necessitate ongoing updates of the standard to accommodate new errors of selection, new clinical data, new and discontinued drugs, and anomalies in the application of Tall Man rules, and to ensure that the selectivity of this approach is maintained. In maintaining the standard, and considering the documented risk of 'alert fatigue'[8], we 
propose that the total number of drug names included in the standard should not vary by more than $\pm 10 \%$ from the original list; alternatively, a risk assessment should be conducted to reprioritise drug names. The Australian Commission on Safety and Quality in Health Care developed and maintains the national standard, including through a notification procedure for errors involving drug name confusion.

Our Australian standard now requires workplace testing and integration into electronic health applications. One opportunity is national standardisation of on-screen presentation of drug names, incorporating Tall Man lettering, to minimise risks of erroneous on-screen drug name selection during prescribing and dispensing. While an international standard would not be feasible, due to variability in drug naming and availability between countries, the derivation processes described here may be adapted by other countries in which prescribing and clinical practice is conducted in English.

\section{CONCLUSION}

The application of Tall Man nomenclature to selected confusable drugs is one initiative to reduce the risk of drug selection errors. The standard reported here was developed via identification of relevant confusable drugs, prioritisation of these drugs based on name similarity and clinical risk, and application of the Mid-Tall Man convention for their name representation. The systematic evaluation enables regular updating of the standard. 


\section{ACKNOWLEDGEMENTS}

The authors acknowledge the assistance of the Tall Man Reference Group, comprising representatives of government, clinical and academic groups. This work was supported by the Clinical Excellence Commission, the Australian Commission for Quality and Safety in Health Care, and The University of Queensland, Australia.

\section{REFERENCES}

1. Peterson, G. M., Wu, M. S. H., Bergin, J. K. (1999) Pharmacists' attitudes towards dispensing errors: their causes and prevention. Journal of Clinical Pharmacy and Therapeutics, 24, 57-71.

2. Kramer, J. M. (1995) More on drug-name confusion. New England Journal of Medicine, 332(11), 754-755.

3. Institute for Safe Medication Practices. (2010) ISMP's List of Confused Drug Names. Available from: http://www.ismp.org/Tools/confuseddrugnames.pdf.

4. Coppock, J. (2007) PDL Risk Management News: In scanners we should trust. Australian Journal of Pharmacy, 88, 12.

5. Food and Drug Administration. (2008) Guidance for Industry: Contents of a Complete Submission for the Evaluation of Proprietary Names. Washington: US Department of Health and Human Services.

6. Food and Drug Administration. (2008) PDUFA Pilot Project: Proprietary Name Review Concept Paper. Washington: US Department of Health and Human Services.

7. Pharmacopeia U. Use Caution - Avoid Confusion. (2001) USP Quality Review, 76. Available from: http://www.usp.org/pdf/EN/patientSafety/qr762001-03-01.pdf.

8. Emmerton, L. M., Rizk, M. F. (2012) Look-alike and sound-alike medicines: risks and 'solutions'. International Journal of Clinical Pharmacy, 34(1), 4-8. 
9. Lambert, B., Yu, C., Thirumalai, M. (2004) A system for multiattribute drug product comparison. Journal of Medical Systems, 28(1), 31-56.

10. Lambert, B., Lin, S., Tan, H. (2005) Designing safe drug names. Drug Safety, 28(6), 495-512. 11. Pharmaceutical Defence Limited. (2008) Ninety-Sixth Annual Report and Statement of Accounts for the year ended 30 June 2008. Hawthorn, Victoria: PDL.

12. Davis, N.M., Cohen, M.R., Teplitsky, B. (1992) Look-alike and sound-alike drug names: the problem and the solution. Hospital Pharmacy, 27(2): 95-110.

13. Ostini, R., Roughead, E. E., Kirkpatrick, C. M., Monteith, G.R., Tett, S.E. (2012) Quality Use of Medicines--medication safety issues in naming; look-alike, sound-alike medicine names. The International Journal of Pharmacy Practice, 20(6), 349-357.

14. Kondrak, G., Dorr, B. (2006) Automatic identification of confusable drug names. Artificial Intelligence in Medicine, 36, 29-42.

15. Lambert, B., Donderi, D., Senders, J. (2002) Similarity of drug names: comparison of objective and subjective measures. Psychology and Marketing, 19(7-8): 641-661.

16. Lambert, B.L. (1997) Predicting look- and sound-alike medication errors. American Journal of Health-System Pharmacy, 54: 1161-1171.

17. Filik, R., Purdy, K., Gale, A., Gerrett, D. (2004) Drug name confusion: evaluating the effectiveness of capital ("Tall Man") letters using eye movement data. Social Science and Medicine, 59: 2597-25601.

18. Filik, R., Purdy, K., Gale, A., Gerrett, D. (2006) Labeling of medicines and patient safety: evaluating methods of reducing drug name confusion. Human Factors, 48(1), 39-47.

19. Gabriele, S. (2006) The role of typography in differentiating look-alike/sound-alike drug names. Healthcare Quarterly, 9(Special Issue), 88-95.

20. Van de Vreede, M., McRae, A., Wiseman, M., Dooley, M. (2008) Successful introduction of Tallman letters to reduce medication selection errors in a hospital network. Journal of Pharmacy Practice and Research, 38(4), 263-236.

21. Gerrett, D., Gale, A., Darker, I. T., FIlik, R., Purdy, K. J. (2009) Final Report of the Use of Tall Man Lettering to Minimise Selection Errors of Medicine Names in Computer Prescribing and 
Dispensing Systems. London: NHS Connecting for Health. Available from:

http://www.connectingforhealth.nhs.uk/systemsandservices/eprescribing/refdocs/tallman.pdf.

22. Emmerton L., Rizk M. (2010) Look-Alike and Sound-Alike Medicines: Reducing the Risk of Errors. Brisbane, Australia: The University of Queensland.

23. National Patient Safety Agency. (2008) A Risk Matrix for Managers. Available from: http://www.nrls.npsa.nhs.uk/resources/?EntryId45=59833.

24. National Coordinating Council for Medication Error Reporting and Prevention. (2001) Index for Categorizing Medication Errors. Available from: http://www.nccmerp.org/pdf/indexBW2001-0612.pdf.

25. Bhargava, A. (2010) strcmp2. Available from: http://webdocs.cs.ualberta.ca/ ab31/strcmp2/ 26. Institute for Safe Medication Practices. (2010) ISMP updates its list of drug name pairs with TALL man letters. ISMP Medication Safety Alert, 15(23), 1-3.

27. Bepko, R., Moore, J., Coleman, J. (2009) Implementation of a pharmacy automation system (robotics) to ensure medication safety at Norwalk hospital. Quality Management in Health Care, $18(2), 103-114$

28. Nanji, K., Cina, J., Patel, N., Churchill, W., Gandhi, T., Poon, E. (2009) Overcoming barriers to the implementation of a pharmacy bar code scanning system for medication dispensing: a case study. Journal of the American Medical Informatics Association, 16(5), 645-650.

29. Poon, E., Cina, J., Churchill, W., Patel, N., Featherstone, E., Rothschild, J., et al. (2006) Medication dispensing errors and potential adverse drug events before and after implementing bar code technology in the pharmacy. Annals of Internal Medicine, 145(6), 426-434.

30. Young, J., Slebodnik, M., Sands, L. (2010) Bar code technology and medication administration error. Journal of Patient Safety, 6(2), 115-120.

31. Teliptsky, B. (1969) Confusing names of drugs. Journal of the American Medical Association, 207(13): 2240.

32. Aronson, J. K. (1994) What's in a brand name? British Medical Journal, 308(6937), 11401141. 
33. Hargett, N. A., Ritch, R., Mardirossian, J., Kass, M. A., Podos, S. M. (1977) Inadvertent substitution of acetohexamide for acetozolamide. American Journal of Ophthalmology, 84(4), 580583.

34. Hoffman, J., Proulx, S. (2003) Medication errors caused by confusion of drug names. Drug Safety, 26(7), 445-452. 


\section{FIGURE LEGENDS}

Figure 1. Risk Matrix

\section{Potential Severity}

Minimum Confusion between the two drugs is unlikely to cause any adverse outcome

Minor Confusion between the two drugs is likely to (or has been documented to)

require increased observations or monitoring to ensure that it does not have an adverse outcome

Moderate Confusion between the two drugs is likely to (or has been documented to) require hospitalisation or transfer to a higher level of care (e.g. transfer to Intensive Care)

Major Confusion between the two drugs is likely to (or has been documented to) cause significant injury such as loss of organ function, or would require an intervention to prevent significant injury

Catastrophic Confusion between the two drugs is likely to (or has been documented to) result in patient death or would require an intervention to sustain life

\section{Likelihood of Confusion}

1 Highly likely

5 Highly unlikely

\section{Risk Category \\ E Extreme \\ $\mathrm{H}$ High \\ M Moderate \\ L Low}

Figure 2. Mid-Tall Man Convention (adapted from [21])

Figure 3. Example of the Application of Tall Man Lettering to a Group 
Table 1. Final Tall Man List (Extract)

\begin{tabular}{|l|l|}
\hline aKIMin & aCLin \\
\hline alDOMET & $\begin{array}{l}\text { alDACTONE } \\
\text { alODORM }\end{array}$ \\
\hline alphaprESS & alphaprIL \\
\hline amARYl & amOXIl \\
\hline amIODAROne & amLODIPIne \\
\hline amLODIPIne & amITRIPTYLIne \\
\hline amITRIPTYLIne & amINOPHYLLIne \\
\hline aPomine & aVomine \\
\hline arATAC & $\begin{array}{l}\text { arOPAX } \\
\text { arABLOC }\end{array}$ \\
\hline
\end{tabular}

